\title{
WILTON RIPPLES IN WEAKLY NONLINEAR MODEL EQUATIONS*
}

\author{
BENJAMIN F. AKERS ${ }^{\dagger}$ AND WENXUAN GAO $\ddagger$
}

\begin{abstract}
Traveling near-bichromatic solutions supported at resonant frequencies are computed in a family of nonlinear model equations. Wilton's expansion is calculated for these solutions to all orders, and used as the basis for a perturbative numerical method for computing near-bichromatic traveling waves. The perturbative method relies on the analyticity of solutions with respect to wave amplitude. Results are compared to a non-perturbative continuation method, and a method for proving analyticity of solutions is proposed.
\end{abstract}

Key words. Water waves, nonlinear waves, resonances.

AMS subject classifications. 76B15, 74J30, 35B34.

\section{Introduction}

The traveling water wave problem has been studied for over a century. In his seminal paper, Stokes derived the weakly nonlinear corrections to nearly monochromatic progressive waves, and more importantly, noted the nonlinear relationship between wave speed and amplitude [1]. Stokes' approach was later expanded to nearly bichromatic waves by Wilton, who noted that two resonant frequencies may travel together only for some amplitude ratios [2]. Both of these historic papers used a perturbative approach to compute the leading order asymptotics of traveling water waves. More recently, such a technique has been used to numerically compute traveling monochromatic water waves and their stability, essentially carrying out the perturbation series to arbitrary order, and invoking the analyticity of the solution $[3,4,5,6]$ or its spectrum $[7,8]$. We develop such a perturbative method for computing ripple-like solutions of a family of weakly nonlinear models, which includes some geophysical wave models. Our numerical method is an example of a high-order spectral method (HOS) [9, 10]. Typically such perturbative methods are either exact in their computations at each perturbation order - but limited to a small number of orders [1, 2], or compute to arbitrary order - but solve for each correction numerically [3, 11]. In this work, the solution at each order is exactly calculated and the calculation has been carried out to arbitrary order, as in [12].

The bichromatic waves we compute include the resonant configuration - 'Wilton's ripple', which is supported at leading order at a wavenumber and its first harmonic. Note that traveling waves which are supported at leading order at a wavenumber and any harmonic are also sometimes referred to as Wilton ripples. In the water wave equations Wilton ripples and similar resonant short-crested waves have been rigorously shown to exist [13], numerically computed [14, 15], and asymptotically approximated $[2,6]$. We compute such bichromatic waves in the family of model equations

$$
u_{t}-L u_{x}+\left(u^{2}\right)_{x}=0
$$

*Received: August 20, 2011; accepted (in revised version): November 21, 2011. Communicated by Peter Smereka.

${ }^{\dagger}$ Department of Mathematics and Statistics, Air Force Institute of Technology, Wright Patterson Air Force Base 45433, USA (Benjamin.Akers@afit.edu).

${ }_{\ddagger}^{\ddagger}$ Department of Computer Science, University of Illinois at Chicago, Chicago, IL 60607, USA. 
The operator $L$ is pseudodifferential, defined via its Fourier symbol $\hat{L u}=\hat{L}(k) \hat{u}(k)$, where $\hat{L}(k)$ is a real, non-monotonic function of the dual variable $k$. Both the computations and numerical results are developed for the general $L$; numerical results are presented for a few choices of $L$ which for which (1.1) has bichromatic solutions. The majority of the numerics are presented for $\hat{L}(k)=\frac{1}{|k|}+\sigma|k|$, in which case Equation (1.1) is a model for deep water gravity capillary water waves, derived in [16] and compared with experiments in [17]. A second choice, $\hat{L}(k)=k^{2}+\sigma k^{4}$, yields the 5th order Korteweg-de Vries equation, sometimes SKdV [18, 19]. Two other examples include the dispersion relations of deep water gravity capillary waves in potential flow $\hat{L}(k)=\left(|k|^{-1}+\sigma|k|\right)^{1 / 2}[20]$ or elastic waves on ice sheets $\hat{L}(k)=\left(|k|^{-1}+\sigma|k|^{3}\right)^{1 / 2}$ [21]. In each case, the parameter $\sigma$ is a constant, playing the role of the Bond number in water waves. The key feature of the operator $L$ is that it must have two copropagating harmonics, $\hat{L}(n k)=\hat{L}(k)$, for integer $n$.

Branches of traveling solutions to models like (1.1) may also be computed via continuation methods. Such an approach typically uses a genuine nonlinear solver, say Newton's method, to compute a traveling wave at each amplitude. Continuation methods have been well developed in the water wave problem $[22,14,23]$ as well as in approximate model equations $[24,25]$. In this work we use the results of such a Fourier collocation/Newton's solver based continuation method [26] as well as a, developed herein, perturbative method similar to $[5,6]$. Both methods have their strengths and weaknesses. The perturbative method relies heavily on analyticity of the solution with respect to some measure of amplitude, $\epsilon$, and will only compute solutions for a range of $\epsilon$ about zero. On the other hand, the pertubative method allows one to know with certainty that nearby waves are part of a smooth branch of solutions by computing not individual points on a curve but the curve itself. The continuation methods strength is in its ability to compute waves at arbitrary amplitude, for example past the radius of convergence of a perturbative method. This method is however, sensitive to its initial guess. Both methods are used here to compute ripple-like traveling wave solutions. Once a branch of such waves is computed, wave statistics such as the dependence of amplitude or energy on speed can also be determined. Such statistics are of particular importance when studying traveling wave stability $[27,28,29]$. Because the expansion is carried out to general order, and the corrections are exactly determined, the numerical method can serve as a foundation from which to prove existence and analyticity of such traveling solutions. As a numerical method it is natural to extend the range of convergence of the perturbation method via a Padé series, which we have also done. Regarding a potential proof of the analyticity of solutions, we expect the Taylor summation to be more useful.

\section{Bichromatic waves and Wilton's ripples}

We consider small amplitude traveling solutions of (1.1), $u=u(x+c t)$, and expand in amplitude, as in $[1,2,3,30]$ :

$$
c=\sum_{q=0}^{\infty} \epsilon^{q} c_{q} \quad \text { and } \quad u=\sum_{q=1}^{\infty} \epsilon^{q} u_{q}(x) .
$$

A solution is referred to as bichromatic if the leading order term $u_{1}$ is supported at two wavenumbers

$$
u_{1}(x)=\left(\beta_{1,1} e^{i k x}+\beta_{1, n} e^{i n k x}\right)+*,
$$

and monochromatic if one $\beta_{1, m}$ is zero. At all orders we choose $u_{q}$ to have zero 


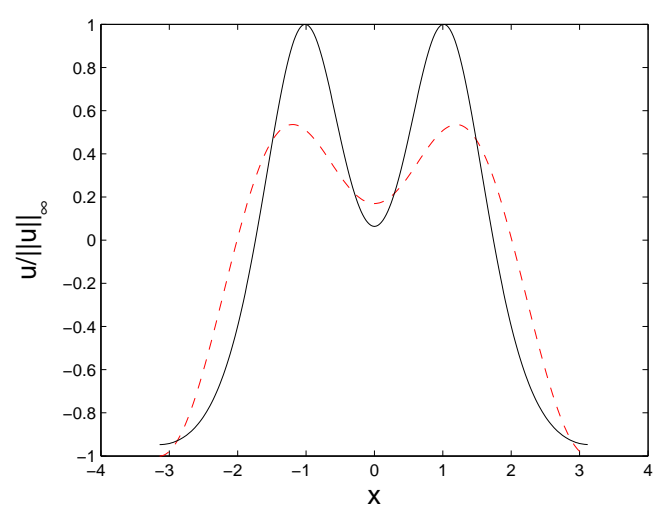

(a)

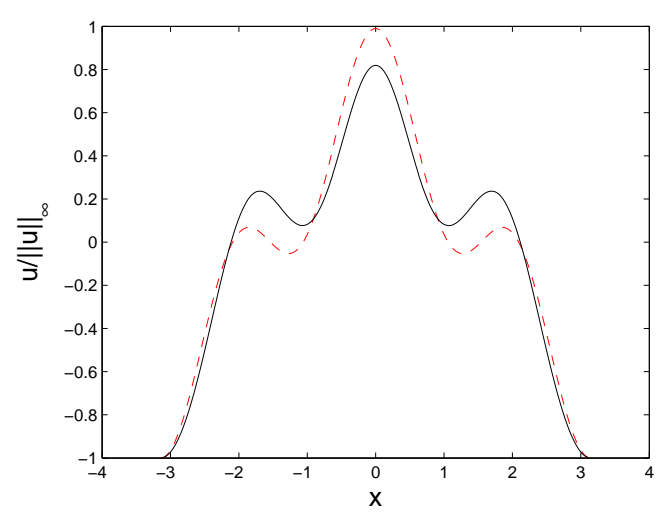

(b)

FIG. 2.1. a) An infinitesimal ripple, $\epsilon=0$ marked with a dashed line compared to a moderate amplitude ripple, $\epsilon=0.1\left(\|u\|_{\infty}=0.245\right)$ marked with a solid line. Both waves are normalized to have unit amplitude in the figure. This branch of waves is marked with triangles pointing down in left panel of Figure 2.2, and is an example of a triad resonance. b) An infinitesimal ripple, $\epsilon=0$ compared to a moderate amplitude ripple, $\epsilon=0.025$ (with $\|u\|_{\infty}=0.0802$ ) marked with a solid line. Both waves are normalized to have unit amplitude in the figure. This branch of waves is marked with circles in the right panel of Figure 2.2, and is an example of a quartet resonance.

mean; a nonzero mean corresponds only to a redefinition of $c$. Thus, in the notation of this paper, a Stokes' wave is monochromatic and Wilton ripples are bichromatic waves. Here and throughout $*$ refers to the complex conjugate of the preceding terms - we only consider real solutions. The parameter $\epsilon$ measures the amplitude of the solution, whose definition we fix by prescribing that $\beta_{1,1}=1$. Notice $\epsilon$ is neither the wave amplitude nor the wave slope, the more traditional definitions. In fact small values of this $\epsilon$ correspond to much larger values of wave amplitude; see Figure 2.1. The character of both the solutions and the perturbation procedure depends critically on the ratio of the wavenumbers, $n$, of the leading order solution. To compute the solution, the ansätze in (2.1) are substituted into (1.1) and the equations at each order are solved, essentially collecting terms at each order from

$$
\left(c_{0}-L+\sum_{q=1}^{\infty} \epsilon^{q} c_{q}\right)\left(\sum_{q=1}^{\infty} \epsilon^{q} u_{q}\right)_{x}+\left(\sum_{q=1}^{\infty} \epsilon^{q} u_{q}\right)\left(\sum_{q=1}^{\infty} \epsilon^{q} u_{q}\right)_{x}=0
$$

and taking care to impose that the equation at each order has a solution which is bounded in both space and time.

The leading order terms from the substitution of (2.1) into (1.1), at $O(\epsilon)$, recover the well known result, $c_{0}=\hat{L}(k)=\hat{L}(n k)$, or that the linear waves move at their respective phase speeds. Notice that for the wave to bichromatic, the phase speed must not be monotonic; although (1.1) includes as special cases the KdV and BenjaminOno equations [31, 32], these equations do not support such bichromatic traveling waves (although they may support more complicated solutions [33]). The phase speed restriction is stronger than non-monotonicity, it must have two copropagating modes where the frequency of the second mode is an integer multiple of the first. This last restriction leads to a complicated problem, finding roots over the integers, which inspired J.R. Wilton to study number theory for the later half of his career [34]. At 
leading order, the amplitudes $\beta_{1,1}$ and $\beta_{1, n}$ are arbitrary — linear theory allows for arbitrary superposition of solutions. Nonlinear solutions, however, exist only about particular values of these amplitudes. For the triad resonant case the choice of value amounts to a sign choice, thus the two branches are sometimes referred to as having a crest dimple or trough dimple. An example of a crest dimple for a triad resonant ripple is in the left panel of Figure 2.1.

At all higher orders, the equation for $u_{q}$ is a forced linear equation of the form

$$
\left(c_{0}-L\right) u_{q, x}=\sum_{m} a_{q, m} e^{i m k x},
$$

where the $a_{q, m}$ are known functions of $u_{1}, \ldots, u_{q-1}$ and $c_{1}, \ldots c_{q}$. For bounded solutions to exist, the coefficients $a_{q, 1}$ and $a_{q, n}$ must vanish, so that the right hand side of (2.3) is orthogonal to the kernel of $\left(c_{0}-L\right)$. These solvability conditions yield two, possibly nonlinear equations at every value of $q$. The form and character of these solvability conditions depends critically on the $n$, the ratio of the leading order frequencies. It is from these solvability conditions that the speed corrections $c_{q}$ and the amplitudes of the second mode in the null space $\beta_{q, n}$ are determined. The form of the solvability conditions can be categorized into two groups: $n=2$ (triad resonances) and $n \geq 3$ (quartet resonances), as noted in [35]. In this work, the triad resonances are between the three wavenumbers $k+k-2 k=0$ with temporal frequencies $\omega(k)+\omega(k)-$ $\omega(2 k)=0$; higher degree resonances have $\mathrm{n}$ instances of wavenumber $k$ summing to wavenumber $n k$ and $n$ temporal frequencies $\omega$ summing to the frequency $\omega(n k)$.

2.1. Triad ripples. Here we present the triad resonance case $(n=2)$. The correction $u_{2}(x)$ is a solution to

$$
\left(c_{0}-L\right) \partial_{x} u_{2}+\sum_{m=-4}^{4} a_{2, m} e^{i m k x}=0,
$$

For this equation to be solvable, with $u_{2}$ bounded (rather than proportionate to $x e^{i x}$ ), the coefficients of $e^{i k x}$ and $e^{2 i k x}$ must vanish, i.e.

$$
a_{2,1}=c_{1} i k+2 i k \beta_{1,2}=0, \quad a_{2,2}=2 c_{1} \beta_{1,2} k+2 k=0 .
$$

The leading order harmonic amplitude, $\beta_{1,2}$, and speed correction, $c_{1}$, are then

$$
\beta_{1,2}= \pm \sqrt{\frac{1}{2}}, \quad c_{1}=\mp \sqrt{2} .
$$

Notice that although $c_{0}$ (and implicitly $k$ ) depend on the linear dispersion relation, these first corrections depend only on the nonlinearity - all choices of $L$ will share the same value of $c_{1}$ and $\beta_{1,2}$. After imposing solvability, we find a particular solution

$$
\begin{aligned}
u_{2, p} & =\beta_{1,2}\left(\frac{a_{2,3}}{\left(\hat{L}(3 k)-c_{0}\right) 3 i k} e^{3 i k x}+\beta_{1,2} \frac{a_{2,4}}{\left(\hat{L}(4 k)-c_{0}\right) 4 i k} e^{4 i k x}\right)+* \\
& \equiv \beta_{2,3} e^{3 i k x}+\beta_{2,4} e^{4 i k x}+*
\end{aligned}
$$

to which we are free to add arbitrary contributions at frequency $k$ and $2 k$; the solution to Equation (2.4) is not unique. We choose the total projection of $u$ onto $e^{i k x}$ to be one, our definition of $\epsilon$. Since this is the value assigned to $\beta_{1,1}$, the Fourier mode of 
the leading order term $u_{1}$, all later orders should have $\beta_{q, 1}=0$. The full correction $u_{2}$ is then

$$
u_{2}=u_{2, p}+\beta_{2,2} e^{2 i k x}+\bar{\beta}_{2,2} e^{-2 i k x} .
$$

Continuing to $O\left(\epsilon^{3}\right)$, the correction $u_{3}$ satisfies the forced equation

$$
\left(c_{0}-\mathcal{L}\right) \partial_{x} u_{3}+\sum_{m=-6}^{6} a_{3, m} e^{i m k x}=0
$$

From this equation the solution $u_{3}=\sum \beta_{3, m} e^{i m k x}$ is determined at all harmonics except $e^{i k x}$ and $e^{2 i k x}$ (and complex conjugates). The coefficient $\beta_{3,2}$ is arbitrary, but will be set by a solvability condition at $O\left(\epsilon^{4}\right)$. The solvability condition at $O\left(\epsilon^{3}\right)$ is

$$
\left(\begin{array}{cc}
1 & 2 \bar{\beta}_{1,1} \\
\beta_{1,2} & c_{1}
\end{array}\right)\left(\begin{array}{c}
c_{2} \\
\beta_{2,2}
\end{array}\right)=-\left(\begin{array}{c}
2 \beta_{2,3} \bar{\beta}_{1,2} \\
2 \beta_{2,4} \bar{\beta}_{1,2}+2 \beta_{2,3} \bar{\beta}_{1,1}
\end{array}\right)
$$

and substituting the values $\beta_{1,1}=1, \beta_{1,2}= \pm \sqrt{\frac{1}{2}}$, and $c_{1}=\mp \sqrt{2}$,

$$
\left(\begin{array}{cc}
1 & 2 \\
\pm \sqrt{\frac{1}{2}} & \mp \sqrt{2}
\end{array}\right)\left(\begin{array}{c}
c_{2} \\
\beta_{2,2}
\end{array}\right)=-\left(\begin{array}{c}
2 \beta_{2,3} \bar{\beta}_{1,2} \\
2 \beta_{2,4} \bar{\beta}_{1,2}+2 \beta_{2,3} \bar{\beta}_{1,1}
\end{array}\right) .
$$

Later orders follow this pattern, at $O\left(\epsilon^{q}\right)$ the solvability conditions are linear in $\beta_{q-1,2}$ and $c_{q-1}$ :

$$
\left(\begin{array}{cc}
1 & 2 \bar{\beta}_{1,1} \\
\beta_{1,2} & c_{1}
\end{array}\right)\left(\begin{array}{c}
c_{q-1} \\
\beta_{q-1,2}
\end{array}\right)=-\left(\begin{array}{c}
2 \beta_{q-1,3} \beta_{1,-2}+\sum_{p=2}^{q-2}\left(\sum_{j=-2 p}^{2 p} \beta_{q-p, 1-j} \beta_{p, j}\right) \\
\sum_{p=1}^{q-1}\left(\sum_{j=-2 p}^{2 p} \beta_{q-p, 2-j} \beta_{p, j}\right)+\sum_{j=2}^{q-2} c_{j} \beta_{q-j, 2}
\end{array}\right) .
$$

The solvability conditions are linear equations for $c_{q}$ and $\beta_{q, 2}$ for $q \geq 3$. The matrix which must be inverted is nonsingular and the same for all $q \geq 3$. The invertibility of this matrix gives formal existence of solutions - we can compute the general term in the series $u_{q}$ and $c_{q}$. We observe the convergence of the series numerically; see panel $\mathrm{b}$ in Figure 2.3. This procedure provides a framework for proofs of both the analyticity and existence of this type of traveling solution; the only missing step is to show that the $c_{q}, u_{q}$ are bounded by $D^{q}$. The growth of the corrections $\left|u_{n}\right|_{\infty}$ is plotted in Figure 2.3. From a least squares fit of $D^{q}$ to these rates, we estimate the radius of convergence of the Taylor sum of the corrections, marked with vertical dashed lines in Figure 2.2. Proving a bound of this type for the corrections $u_{n}$ is a topic of current research. Notice that in Figure 2.2, as often the case, the Taylor sum fails to converge before there is any apparent loss of analyticity, however the applicability of the perturbation procedure can be extended using Padé summation. As a method of proving analyticity with respect to amplitude we expect the Taylor sum to be simpler; as a numerical method to estimate the radius of analyticity one should use the first non-canceled pole of the Padé sum, as in [7].

Two branches of Wilton ripples were computed for (1.1) with $\hat{L}(k)=1 /|k|+|k| / 2$, $\sigma=1 / 2$. These two branches bifurcate from an infinitesimal linear wave supported at wavenumbers $k=1$ and 2 , moving at speed $c_{0}=3 / 2$. The two branches correspond to the two sign choices for $c_{1}= \pm \sqrt{2}$. The dependence of the speed on $\epsilon$ is plotted in Figure 2.2. Examples of infinitesimal and moderate amplitude triad ripple profiles are in the left panel of Figure 2.1. 


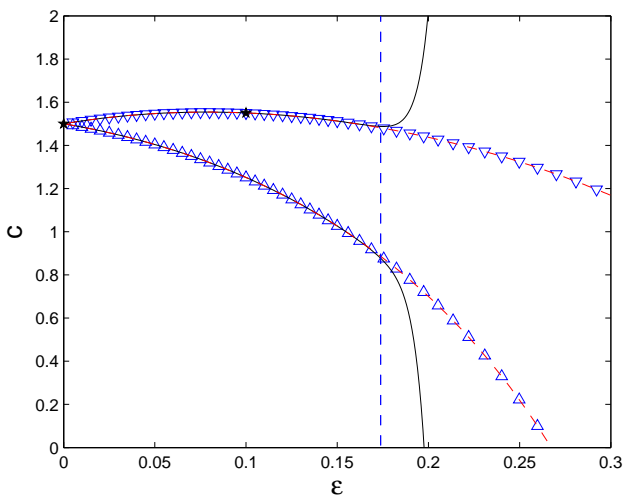

(a)

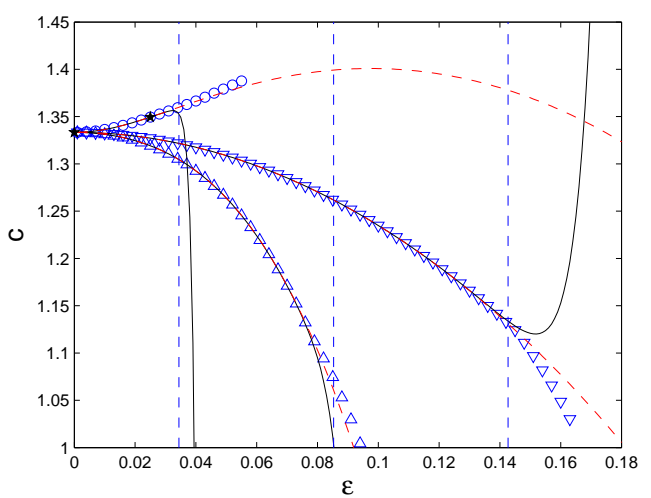

(b)

FIG. 2.2. a) The speed of two branches of Wilton ripples are plotted as a function of the magnitude of the Fourier mode $k=1$, our definition of $\epsilon$. Solid curves mark the results of the perturbative numerical method; triangles are the results of the Fourier collocation/Newton's method, dashed curves mark a Padé approximation. The wave whose profiles are in Figure 2.1 is marked with solid star. The dashed vertical line which marks the estimated radius of convergence of the perturbative method, using a Taylor summation, at $\epsilon \approx 0.17$. The first un-canceled pole of the Padé sum gives estimates of $\epsilon \approx 1.12$ for the upper branch and $\epsilon \approx 0.96$ for the lower one. $b)$ The speeds of three branches of quartet-resonant traveling waves are plotted as a function of the magnitude of the Fourier mode $k=1$, here defined as $\epsilon$. The curves are marked as in the right panel. The estimated radii of convergence of the perturbative method for each branch are $\epsilon=0.034,0.085$, and 0.14 ; the first uncanceled poles of the appropriate Padé sum are at $\epsilon \approx 1.6,2.2$, and 3.7.

2.2. Quartets and bichromatic waves. For $n \geq 3$ there are no triad interactions and the $O\left(\epsilon^{2}\right)$ solvability conditions imply that $c_{1}=0$ while leaving $\beta_{1, n}$ arbitrary. In this section we present the details of the series for $n=3$, highlighting the differences from the, very similar, general case $(n>3)$ along the way. The quartet solutions are still supported at leading order at two frequencies, now $k=1$ and $k=3$. The nonlinear solvability condition which determines $\beta_{1,3}$ is now due to quartet resonances:

$$
\begin{array}{r}
c_{2}+\alpha_{1}\left|\beta_{1,3}\right|^{2}+\alpha_{2} \beta_{1,3}+\alpha_{3}=0 \\
\beta_{1,3} c_{2}+\alpha_{4} \beta_{1,3}+\alpha_{5}+\alpha_{6}\left|\beta_{1,3}\right|^{2} \beta_{1,3}=0,
\end{array}
$$

where

$$
\begin{gathered}
\alpha_{1}=\alpha_{4}=4\left(\frac{1}{\hat{L}(4 k)-c_{0}}+\frac{1}{\hat{L}(2 k)-c_{0}}\right), \quad \alpha_{2}=\frac{6}{\hat{L}(2 k)-c_{0}}, \\
\alpha_{3}=\alpha_{5}=\frac{2}{\hat{L}(2 k)-c_{0}}, \quad \alpha_{6}=\frac{2}{\hat{L}(6 k)-c_{0}} .
\end{gathered}
$$

We assume that $\left(c_{0}-\hat{L}(m k)\right)$ is nonzero for $m \notin\{1, n\}$, so that the $\alpha_{j}$ are finite, essentially assuming on a periodic domain the operator $\left(c_{0}-L\right)$ has a two-dimensional kernel. Since the speed $c_{2}$ is real, Equation (2.6a) implies that $\beta_{1,3}$ is real, essentially a consequence of the phase choice we make by setting $\beta_{1,1}=1$ instead of $\left|\beta_{1,1}\right|=1$. This cubic system for $\left(\beta_{1,3}, c_{2}\right)$ must generically be solved numerically (this is the 
only numerical approximation of the method apart from truncation after some value of $q)$. Given a solution of $(2.6)\left(\beta_{1,3}, c_{2}\right)$, we can compute a branch of solutions to (1.1). For $q>3$, the solvability conditions are linear in $\beta_{q-2,3}$ and $c_{q-1}$. For even $q$ they are

$$
\left(\begin{array}{cc}
\beta_{1,1} & 2\left(\beta_{2,-2}+\beta_{2,4}\right) \\
\beta_{1,3} & c_{2}+2 \beta_{2,6}
\end{array}\right)\left(\begin{array}{c}
c_{q-1} \\
\beta_{q-2,3}
\end{array}\right)=\left(\begin{array}{c}
F_{1} \\
F_{2}
\end{array}\right)
$$

with

$$
F_{1}=-\sum_{p=4}^{q-4}\left(\beta_{q-p,-2} \beta_{p, 3}+\beta_{q-p, 4} \beta_{p,-3}\right), \quad F_{2}=-\sum_{p=3}^{q-3} \beta_{q-p, 6} \beta_{p,-3},
$$

while for odd $q$ they are

$$
\begin{aligned}
& \left(\begin{array}{cc}
\beta_{1,1} & 2\left(\beta_{2,-2}+\beta_{2,4}\right)+\frac{4}{\hat{L}(2 k)-c_{0}}+\frac{4}{\hat{L}(4 k)-c_{0}} \beta_{1,-3}+\frac{4}{\hat{L}(2 k)-c_{0}} \beta_{1,3} \\
\beta_{1,3} & c_{2}+2 \beta_{2,6}+\frac{4}{\hat{L}(2 k)-c_{0}}+\frac{4}{\hat{L}(4 k)-c_{0}}+\frac{4}{\hat{L}(6 k)-c_{0}} \beta_{1,3} \beta_{1,-3}
\end{array}\right)\left(\begin{array}{c}
c_{q-1} \\
\beta_{q-2,3}
\end{array}\right) \\
= & \left(\begin{array}{c}
F_{3} \\
F_{4}
\end{array}\right)
\end{aligned}
$$

with

$$
\begin{aligned}
& F_{3}=-\left(2 \sum_{\substack{j=-6 \\
j \neq 1 \pm 3}}^{6} \beta_{q-2,1-j} \beta_{2, j}+\sum_{p=3}^{q-3} \sum_{j=-3 p}^{3 p} \beta_{q-p, 1-j} \beta_{p, j}\right. \\
& +\frac{4 \sum_{j \in K_{1}, j \neq-1} \beta_{q-2,2-j} \beta_{1, j}+\sum_{p=2}^{q-3} 2 \sum_{j=-3 p}^{3 p} \beta_{q-1-p, 2-j} \beta_{p, j}+2 \sum_{j=2}^{q-3} c_{j} \beta_{q-1-j, 2}}{\hat{L}(2 k)-c_{0}} \\
& +\frac{4 \sum_{j \in K_{1}, j \neq 1} \beta_{q-2,4-j} \beta_{1, j}+\sum_{p=2}^{q-3} 2 \sum_{j=-3 p}^{3 p} \beta_{q-1-p, 4-j} \beta_{p, j}+2 \sum_{j=2}^{q-3} c_{j} \beta_{q-1-j, 4}}{\hat{L}(4 k)-c_{0}} \beta_{1,-3} \\
& \left.+\frac{4 \sum_{j \in K_{1}, j \neq-1} \beta_{q-2,2-j} \beta_{1, j}+\sum_{p=2}^{q-3} 2 \sum_{j=-3 p}^{3 p} \beta_{q-1-p, 2-j} \beta_{p, j}+2 \sum_{j=2}^{q-3} c_{j} \beta_{q-1-j, 2}}{\hat{L}(2 k)-c_{0}} \beta_{1,3}\right)
\end{aligned}
$$

and

$$
\begin{aligned}
F_{4}= & -\left(\sum_{j=3}^{q-2} c_{j} \beta_{q-j, 3}+2 \sum_{\substack{j=-6, j \neq 3 \pm 3}}^{6} \beta_{q-2,3-j} \beta_{2, j}+\sum_{p=3}^{q-3} \sum_{j=-3 p}^{3 p} \beta_{q-p, 3-j} \beta_{p, j}\right. \\
& +\frac{4 \sum_{j \in K_{1}, j \neq-1} \beta_{q-2,2-j} \beta_{1, j}+\sum_{p=2}^{q-3} 2 \sum_{j=-3 p}^{3 p} \beta_{q-1-p, 2-j} \beta_{p, j}+2 \sum_{j=2}^{q-3} c_{j} \beta_{q-1-j, 2}}{\hat{L}(2 k)-c_{0}}
\end{aligned}
$$




$$
\begin{aligned}
& +\frac{4 \sum_{j \in K_{1}, j \neq 1} \beta_{q-2,4-j} \beta_{1, j}+\sum_{p=2}^{q-3} 2 \sum_{j=-3 p}^{3 p} \beta_{q-1-p, 4-j} \beta_{p, j}+2 \sum_{j=2}^{q-3} c_{j} \beta_{q-1-j, 4}}{\hat{L}(4 k)-c_{0}} \\
& \left.+\frac{4 \sum_{j \in K_{1}, j \neq 3} \beta_{q-2,6-j} \beta_{1, j}+\sum_{p=2}^{q-3} 2 \sum_{j=-3 p}^{3 p} \beta_{q-1-p, 6-j} \beta_{p, j}+2 \sum_{j=2}^{q-3} c_{j} \beta_{q-1-j, 6}}{\hat{L}(6 k)-c_{0}} \beta_{1,-3}\right) .
\end{aligned}
$$

In summary, for $n=3$ a cubic system is solved for $\beta_{1,3}$ and $c_{2}$, where all the later pairs $\left(\beta_{q-1,3} c_{q}\right)$ are determined from linear equations. For $n>3$, two of the terms in (2.6) do not appear, and the nonlinear solvability conditions are

$$
\begin{array}{r}
c_{2}+\gamma_{1}\left|\beta_{1, n}\right|^{2}+\gamma_{2}=0, \\
\beta_{1, n}\left(c_{2}+\gamma_{1}+\gamma_{3}\left|\beta_{1, n}\right|^{2}\right)=0,
\end{array}
$$

with

$$
\begin{aligned}
\gamma_{1} & =\left(\frac{4}{\hat{L}(2(n+1) k)-c_{0}}+\frac{4}{\hat{L}(2(n-1) k)-c_{0}}\right), \\
\gamma_{2} & =\frac{2}{\hat{L}(2 k)-c_{0}}, \quad \text { and } \quad \gamma_{3}=\frac{2}{\hat{L}(2 n k)-c_{0}} .
\end{aligned}
$$

The cubic (2.9) is exactly solvable with generically a single solution pair $\beta_{1, n}=0$ and $c_{2}=-\gamma_{2}$. A pathological scenario arises should $\gamma_{1}=\gamma_{2}=\gamma_{3}$, which we do not focus on here. In the general case, where $\gamma_{1}, \gamma_{2}$, and $\gamma_{3}$ are not equal, the solution for $n>3$ is a Stoke's wave (which is supported at leading order only at one frequency). As in the $n=3$ case, for $n>3$ all solvability conditions for $q>3$ are linear, similar in form to equations (2.7) and (2.8).

Three branches of bichromatic waves were computed to Equation (1.1) with $\hat{L}(k)=1 /|k|+|k| / 3$, setting $\sigma=1 / 3$. These two branches bifurcate from an infinitesimal linear wave supported at wavenumbers $k=1$ and 3 , moving at speed $c_{0}=4 / 3$. The three branches correspond to the three roots of the cubic in Equation (2.6). The dependence of the speed on $\epsilon$ is plotted in the right panel of Figure 2.2; examples of an infinitesimal and moderate amplitude quartet ripple are in the right panel of Figure 2.1.

Of especial interest in Figure 2.2 is the radius of convergence of the perturbative method, marked with vertical dotted lines in panel a. These numerically estimated radii mark the boundary after which the Taylor summation, derived here, diverges. For the example presented here, the traveling waves appear to smoothly continue past these boundaries, and a Padé summation is used to extend the perturbation method. To numerically approximate the disc of analyticity it would then be useful to use the first non-canceled pole of the Padé sum, rather than the growth rate of the Taylor sum. In pursuing a proof of analyticity of solutions, we plan to use the Taylor sum, as we have general formulae for the corrections (for example (2.5)). Whether or not there is some loss of analyticity of the solution branch, for example a secondary bifurcation, at either critical values (Taylor or Padé) of $\epsilon$ is an open question.

\section{Conclusion}

A perturbative method is presented for computing nearly bichromatic waves in a family of model equations. This method computes the Taylor coefficients for analytic 


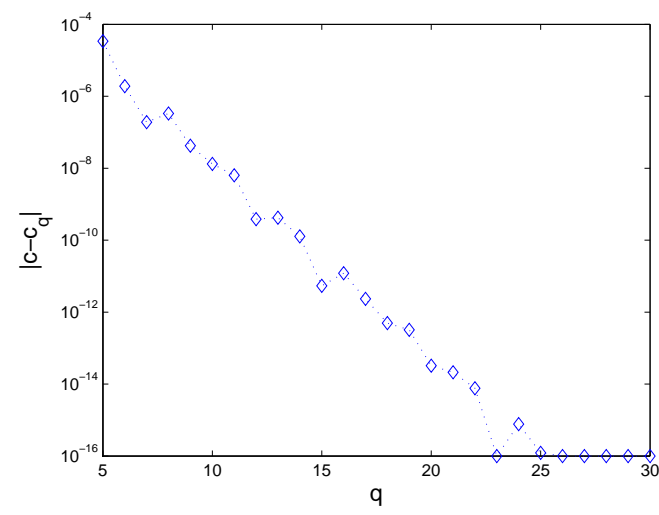

(a)

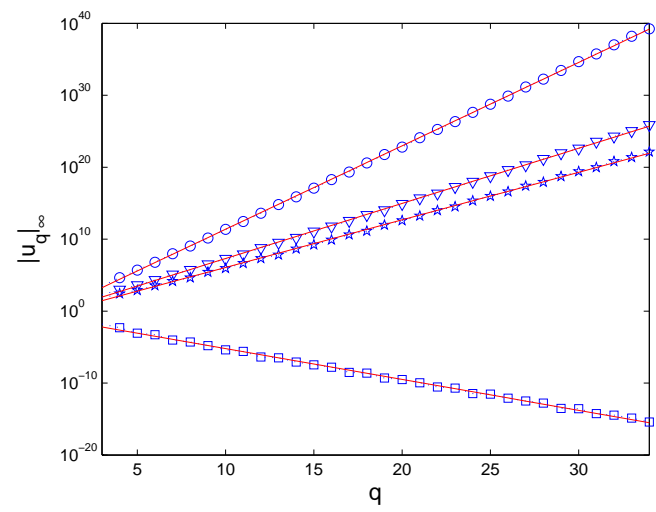

(b)

FIG. 2.3. a) The convergence of the speed $c_{q}$ to the wave speed $c$ (computed with the nonperturbative Newton solver/Fourier Collocation method), is plotted as a function of the perturbation order $q$ at $\epsilon=0.05$, for the model Equation (1.1) with $\hat{L}=|k|^{-1}+|k|$. b) The growth rate of the corrections $\left\|u_{n}\right\|_{\infty}$ for four choices of the linear operator: $\hat{L}=\sqrt{|k|^{-1}+|k|}$ marked with circles, $\hat{L}=|k|^{-1}+|k|$ marked with triangles, $\hat{L}=\sqrt{|k|^{-1}+|k|^{3}}$ marked with stars, and $\hat{L}=k^{2}-\frac{3}{15} k^{4}$ marked with squares. In each case, the growth rate is approximated by $D^{k}$, the solid lines, with $D \approx 14.4,5.8,4.8$, and 0.37 respectively.

branches of traveling solutions, and provides a framework from which to prove existence and analyticity. The method is compared to a continuation procedure based on Fourier-collocation and Newton's method, and can be extended with Padé summation. Branches of solutions are computed for an example model equation, including both wave profiles and the waves' speed-amplitude relationships. The disc of analyticity is numerically estimated and a framework for proving analyticity is provided. The stability of these traveling waves is an open question, wherein the perturbative method may be especially useful.

\section{REFERENCES}

[1] G.G. Stokes, On the theory of oscillatory waves, Trans. Camb. Phil. Soc., 8, 441-455, 1847.

[2] J.R. Wilton, On ripples, Phil. Mag., 29, 173, 1915.

[3] L.W. Schwartz, Computer extension and analytic continuation of Stokes' expansion for gravity waves, J. Fluid Mech., 62, 553-578, 1974.

[4] D.P. Nicholls and F. Reitich, On analyticity of traveling water waves, Proc. Roy. Soc. Lond., A, 461(2057), 1283-1309, 2005.

[5] D.P. Nicholls and F. Reitich, Stable, high-order computation of traveling water waves in three dimensions, Eur. Jour. Mech. B/ Fluids, 25, 406-424, 2006.

[6] B. Akers and D.P. Nicholls, Traveling water waves with gravity and surface tension, SIAM J. Appl. Math., 70, 2373-2389, 2010.

[7] D.P. Nicholls, Spectral data for traveling water waves: Singularities and stability, J. Fluid Mech., 624, 339-360, 2009.

[8] B. Akers and D.P. Nicholls, Spectral stability of deep two-dimensional gravity water waves: Repeated eigenvalues, SIAM J. Appl. Math., accepted, 2012.

[9] W.T. Tsai and D.K.P. Yue, Computation of nonlinear free-surface flows, Ann. Rev. Fluid Mech., 28, 249-278, 1996.

[10] D.P. Nicholls, Boundary perturbation methods for water waves, GAMM-Mitt., 30, 44-74, 2007.

[11] D.G. Dommermuth and D.K.P. Yue, A high-order spectral method for the study of nonlinear gravity waves, J. Fluid Mech., 184, 267-288, 1987. 
[12] S.E. Haupt and J.P. Boyd, Modeling nonlinear resonance: A modification to the stokes' perturbation expansion, Wave Motion, 10, 83-98, 1988.

[13] J. Reeder and M. Shinbrot, Three dimensional, nonlinear wave interaction in water of constant depth, Nonlin. Anal., 5, 303-323, 1981.

[14] O. Kimmoun, H. Branger, and C. Kharif, On short-crested waves: Experimental and analytical investigations, Eur. J. Mech. B/Fluids, 18, 889-930, 1999.

[15] J.M. Vanden-Broeck, Wilton ripples generated by a moving pressure disturbance, J. Fluid Mech., 451, 193-201, 2002.

[16] B. Akers and P.A. Milewski, A model equation for wavepacket solitary waves arising from capillary-gravity flows, Stud. Appl. Math., 122, 249-274, 2009.

[17] Y.-W. Cho, J.D. Diorio, T.R. Akylas, and J.H. Duncan, Resonantly forced gravity-capillary lumps on deep water. Part 2. Theoretical model, J. Fluid Mech., 672, 288-306, 2011.

[18] S.E. Haupt and J.P. Boyd, Double cnoidal waves of the Korteweg-de Vries equation: A boundary value approach, Physica D, 50, 117-134, 1991.

[19] B. Malomed and J.M. Vanden-Broeck, Solitary wave interactions in the 5th order KdV equation, Contemp. Math., 200, 133-143, 1996.

[20] F. Dias and C. Kharif, Nonlinear gravity and gravity-capillary waves, Ann. Rev. Fluid Mech., 31, 301-346, 1999.

[21] E. Parau and F. Dias, Nonlinear effects in the response of a floating ice plate, J. Fluid Mech., 460, 281-305, 2002.

[22] D.I. Meiron, P.G. Saffman, and H.C. Yuen, Calculation of steady three dimensional deep-water waves, J. Fluid Mech., 124, 109-121, 1982.

[23] J.M. Vanden-Broeck and F. Dias, Gravity-capillary solitary waves in water of infinite depth and related free surface flows, J. Fluid Mech., 240, 549-557, 1992.

[24] B. Akers and P.A. Milewski, Dynamics of three dimensional gravity-capillary waves in deep water, SIAM J. Appl. Math., 70, 2390-2408, 2010.

[25] B. Kim and T.R. Akylas, On gravity-capillary lumps, J. Fluid Mech., 540, 337-351, 2005.

[26] B. Akers and P.A. Milewski, Model equations for gravity-capillary waves in deep water, Stud. Appl. Math., 121, 49-69, 2008.

[27] B. Akers and P.A. Milewski, A stability result for travelling waves in dispersive nonlinear equations, Commun. Math. Sci., 6, 791-797, 2008.

[28] D.P. Bennett, R.W. Brown, S.E. Stansfield, J.D. Stroughair, and J.L. Bona, The stability of internal solitary waves, Math. Proc. Camb. Phil. Soc., 94, 351-379, 1983.

[29] P.G. Saffman, The superharmonic instability of finite amplitude water waves, J. Fluid Mech., $159,169-174,1985$.

[30] W.J. Harrison, The influence of viscosity and capillarity on waves of finite amplitude, Proc. Lond. Math. Soc., 7, 107-121, 1909.

[31] H. Ono, Algebraic solitary waves in stratified fluids, J. Phys. Soc. Japan, 39(4), 1082-1091, 1975.

[32] D.J. Korteweg and G. de Vries, On the change in form of long waves advancing in a rectangular canal and new type of long stationary waves, Phil. Mag., 39(5), 422-443, 1895.

[33] J.P. Boyd, Weakly Nonlocal Solitary Waves and Beyond-All-Orders Asymptotics : Generalized Solitons and Hyperasypmtotic Perturbation Theory, Mathematics and Its Applications, Kluwer, 442, 1998.

[34] H.S. Carlaw and G.H. Hardy, John Raymond Wilton, J. London Math. Soc., s1-20, 58-64, 1945.

[35] B. Chen and P.G. Saffman, Steady gravity-capillary waves on deep water. I - Weakly nonlinear waves, Stud. Appl. Math., 60, 183-210, 1979. 